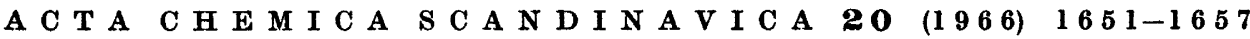

\title{
Separation of Unsaturated Fatty Acids of Soybean and Linseed Oils by Crystallization and Subsequent
} Liquid-liquid Extraction

\author{
ERKKI UKSILA, MATTI VARESMAA and INKERI LEHTINEN
}

Department of Nutritional Chemistry, University of Helsinki, Helsinki, Finland

\begin{abstract}
Soybean and linseed oil fatty acids, first crystallized at $-40^{\circ} \mathrm{C}$ from methanol, were distributed in a 200 -tube countercurrent extraction apparatus with hexane as the mobile top phase. For soybean oil acids a mixture of methanol:furfural:water $(75: 20: 5 \mathrm{v} / \mathrm{v})$ was used as the immobile bottom phase; in this solvent system the distribution coefficients for oleic, linoleic, and linolenic acids were 1.5, 1.1, and 0.65 , respectively. For linseed oil acids a mixture methanol:dimethyl sulfoxide:water $(75: 20: 5 \mathrm{v} / \mathrm{v})$ was used as immobile bottom phase and the distribution coefficients for oleic, linoleic, and linolenic acids were $1.25,0.74$, and 0.51 , respectively.

Linseed oil acids were first crystallized as "acid" sodium soaps from methanol and the fatty acids of the liquid fraction were subjected to continuous separation in a 60-tube countercurrent extraction apparatus. Small successive volumes of the two phases (hexane and methanol:dimethyl sulfoxide:water) were run alternately in opposite directions. About $85 \%$ of the linolenic acid emerging with the effluents was recovered in the bottom phase, its purity being $96.8 \%$ as determined by gas chromatography.
\end{abstract}

iquid-liquid extraction methods are in wide use for the separation of Ldifferent types of material, e.g. fatty acids, for analytical purposes and in small-scale work (see Ref. 4). For the industrial production of pure fatty acids they are not employed so far, but they seem to be quite promising also for large-scale production of reasonable pure fatty acids. ${ }^{2,3,7}$

For separating linoleic acid and linolenic acids from soybean and linseed oils in the purity of at least $90 \%$, crystallization methods are not sufficiently effective. $^{8}$ However, as a procedure preceding liquid-liquid extraction, crystallization might be successful for the preliminary purification of fatty acids. The present paper describes experiments made to use these two methods as combined. 


\section{METHODS}

The fatty acids of soybean and linseed oils were prepared by the alkali saponification method described previously. ${ }^{8}$

The fatty acid analyses were performed by a PYE argon chromatograph with a poly. ester succinate (BDS) column. ${ }^{8}$

Countercurrent extraction. Two automatic countercurrent extraction apparatuses with a tube model developed by Hietala ${ }^{6}$ were used; the respective tube volumes were 13.7 and $14.1 \mathrm{ml}$. The emerging of the fatty acids in the effluents was followed by titration: samples of $2 \mathrm{ml}$ were mixed with $4 \mathrm{ml}$ of $95 \%$ ethanol and titrated with standard sodium hydroxide using phenolphthalein as the indicator. Nitrogen gas was led through the solution to stir it effectively during titration. The fatty acid composition of the effluent fractions was determined by gas chromatography.

Two solvent systems were employed, one consisting of hexane as the lighter phase and methanol:furfural:water $(75: 20: 5 \mathrm{v} / \mathrm{v})$ as the heavier phase; in the other system furfural was replaced by dimethyl sulfoxide.

\section{RESULTS}

1. Distribution of soybean oil fatty acids. The solvent system consisted of hexane as the mobile top phase and methanol:furfural:water (75:20:5 v/v) as the immobile bottom phase. Methanol (Merck) and hexane (BDH) were used as such without further purification, whereas furfural (Riedel-De Haën) was purified by distilling in vacuo.

The two phases were mutually saturated before they were fed in. The volume of hyperphase in one tube was $4.3 \mathrm{ml}$ and that of hypophase, $9.4 \mathrm{ml}$. The total number of tubes was 200.

A solution containing $40 \%$ soybean oil fatty acids in absolute methanol was crystallized at $-40^{\circ} \mathrm{C}$ for $24 \mathrm{~h}$. The first four distribution tubes were loaded with approximately $5 \mathrm{~g}$ of the fatty acids of the liquid fraction. The acids were distributed allowing the mobile phase to flow in at an approximate rate of $3 \mathrm{ml} / \mathrm{min}$. The effluent hyperphase was collected in fractions of $12 \mathrm{ml}$. The maximum concentrations of oleic, linoleic, and linolenic acids emerged from the apparatus with 2050,2590 , and $3750 \mathrm{ml}$ hyperphase, respectively. The purity of linoleic acid in the combined fractions corresponding to the yield of $95.8 \%$ was $91.1 \%$ as determined by gas chromatography. In addition, it contained $4.3 \%$ oleic acid and $4.6 \%$ linolenic acid.

Distribution coefficients were calculated from the equation ${ }^{5}$

$$
K=\frac{N V_{\mathrm{b}}}{v_{\mathrm{t}}-N V_{\mathrm{t}}}
$$

in which $N=$ serial number belonging to the medium tube of the distribution.

$v_{\mathrm{t}}=$ volume of effluent top phase corresponding to maximum concentration of substance.

$V_{\mathrm{t}}=$ volume of top phase in one distribution tube.

$V_{\mathrm{b}}=$ volume of bottom phase in one distribution tube.

The distribution coefficients of the acids and the separation factors $(\beta)$ were:

$$
\begin{aligned}
& K_{\text {oleic }}=1.5 \\
& K_{\text {linoleic }}=1.1 \\
& K_{\text {linolenic }}=0.65
\end{aligned}
$$$$
\begin{array}{ll}
\beta_{\text {oleic/linoleic }} & =1.4 \\
\beta_{\text {oleic/linolenic }} & =2.3 \\
\beta_{\text {linoleic/linolenic }} & =1.7
\end{array}
$$ 
It is possible to calculate the number of tubes required $\left(X_{t}\right)$ for a desired yield and purity when separating them by countercurrent extraction. ${ }^{5}$ In the above experiment, where the top phase was mobile, the $X_{t}$-values for a $99.9 \%$ yield and a contamination of $1.22 \%$ were as follows:

$\begin{array}{lr}\text { Fatty axid pair } & X_{\mathrm{t}} \\ \text { oleic-linoleic } & 1025 \\ \text { oleic-linolenic } & 126 \\ \text { linoleic-linolenic } & 273\end{array}$

2. Distribution of linseed oil fatty acids. Linseed oil fatty acids were first crystallized at $-40^{\circ} \mathrm{C}$ as described above in connection with soybean oil experiments. The liquid fraction consisted of traces of palmitic acid, $11.9 \%$ oleic, $18.7 \%$ linoleic, and $69.5 \%$ linolenic acid. About $10 \mathrm{~g}$ of this fatty acid mixture was loaded in the first eight distribution tubes of the apparatus.

The solvent system used was hexane-methanol:dimethyl sulfoxide: water $(75: 20: 5 \mathrm{v} / \mathrm{v})$. The experimental conditions were the same as in the soybean oil experiment.

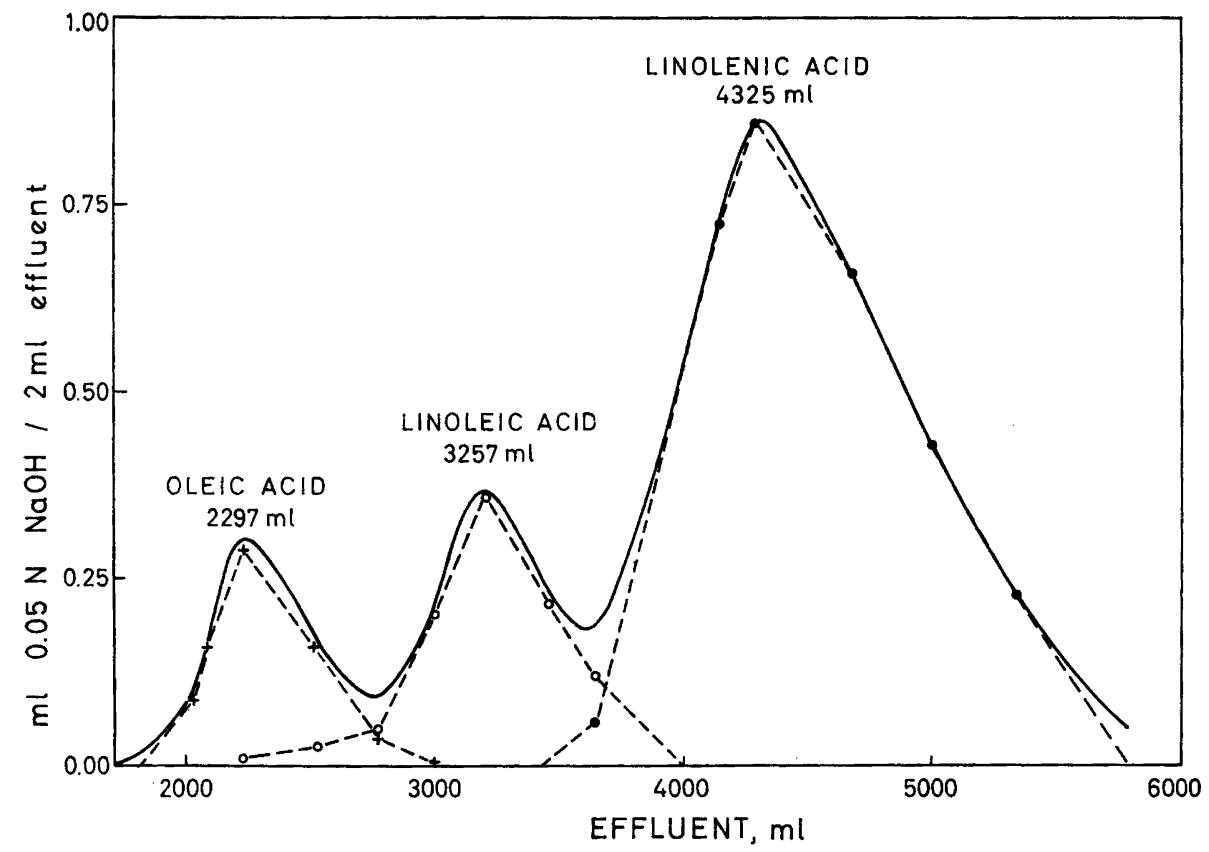

Fig. 1. Countercurrent distribution of linseed oil fatty acids first crystallized from methanol at $-40^{\circ} \mathrm{C}$. Solid line: distribution curve obtained by titration. Dotted lines: distribution curves obtained for each acid as calculated from the titration values according to the gas chromatographic analyses. Tube number: 200. Solvent system: hexane (4.7 $\mathrm{ml} /$ tube) as the mobile hyperphase, methanol:dimethyl sulfoxide:water $(75: 20: 5 \mathrm{v} / \mathrm{v})$ $(8.8 \mathrm{ml} /$ tube) as the immobile hypophase.

Acta Chem. Scand. 20 (1966) No. 6 
Fig. 1 presents the distribution curves of oleic, linoleic, and linolenic acids as well as other details of the results obtained. The distribution curve is somewhat skewed, probably due to the association of the acids.

The distribution coefficients of oleic, linoleic, and linolenic acids were 1.25 , 0.74 , and 0.51 , respectively. The separation factors $(\beta)$ and the $X_{t}$-numbers were as follows:

$\begin{array}{lcr}\text { Fatty acid pair } & \beta & X_{\mathrm{t}} \\ \text { oleic-linoleic } & 1.7 & \mathbf{1 4 7} \\ \text { oleic-linolenic } & 2.5 & \mathbf{3 5} \\ \text { linoleic-linolenic } & \mathbf{1 . 5} & \mathbf{2 5 9}\end{array}$

3. Fractionation by the crystallization-countercurrent extraction process. Linseed oil fatty acids were first crystallized as "acid" sodium soaps and the linolenic acid concentrate thus obtained was subjected to a continuous liquid-liquid extraction procedure in a 60-tube apparatus.

Crystallization as "acid" sodium soaps. Linseed oil acids were dissolved in methanol containing $10 \%(\mathrm{w} / \mathrm{w})$ water. Half of the acids were neutralized with $1 \mathrm{~N} \mathrm{NaOH}$ in aqueous (10\%) methanol and diluted to a solution containing $10 \%(\mathrm{w} / \mathrm{w})$ of "acid" soaps in methanol-water $(90: 10 \mathrm{w} / \mathrm{w})$. The solution was gently warmed to dissolve the "acid" soaps, then cooled to $+6^{\circ} \mathrm{C}$ and filtrated after a few hours. The filtrate was kept over night at $-25^{\circ} \mathrm{C}$. The liquid part was removed by suction through a glass sinter. The yield of the fatty acids in the filtrate was $52 \%$ of the starting amount, and it contained $0.1 \%$ palmitic, less than $1 \%$ palmito-oleic, $11.8 \%$ oleic, $19.8 \%$ linoleic, and $68.2 \%$ linolenic acid.

In order to release the fatty acids, $10 \mathrm{~N} \mathrm{H}_{2} \mathrm{SO}_{4}$ was added to the filtrate to lower the $\mathrm{pH}$ to 6.2. The sodium sulfate precipitate was removed by filtration. Dimethyl sulfoxide was added to the filtrate obtained until the ratio of $\mathrm{MeOH}$ to DMS was 75:20 (v/v). After saturation with hexane this solution was subjected to a liquid-liquid extraction procedure.

Countercurrent extraction. An apparatus with 60 tubes was used. The solvent system consisted of hexane and methanol:dimethyl sulfoxide:water $(75: 20: 5 \mathrm{v} / \mathrm{v})$.

Small successive amounts of both phases were run alternately in opposite directions. The ratio of the influents $\left(v_{\mathrm{t}} / v_{\mathrm{b}}\right)$ required for symmetrical distribution of linoleic and linolenic acids around the tube through which the fatty acids were fed in was calculated from the equation

$$
\left(x_{\mathrm{t}}-x_{\mathrm{b}}\right)_{\text {linoleic }}=\left(x_{\mathrm{t}}-x_{\mathrm{b}}\right)_{\text {linolenic }}
$$

in which $x_{\mathrm{t}}$ and $x_{\mathrm{b}}$ refer to the number of tubes through which the maximum concentrations have passed with the volumes $v_{\mathrm{t}}$ and $v_{\mathrm{b}}$ of top and bottom phases, respectively. In the apparatus used $x_{\mathrm{t}}=v_{\mathrm{t}} /\left(V_{\mathrm{t}}+V_{\mathrm{b}} / K\right)$ and $x_{\mathrm{b}}=v_{\mathrm{b}}\left(V_{\mathrm{b}}+K V_{\mathrm{b}}\right) .^{5}$

Experimental procedure. The liquid-liquid extraction procedure of linseed oil acids first fractionated as "acid" sodium soaps is seen in detail in Fig. 2. The fatty acid solution was fed in with a piston burette simultaneously with the first $100 \mathrm{ml}$ of the bottom phase flowing in. This operation was repeated each time bottom phase was fed in.

The operations required for feeding of fatty acids, alternating the phases flowing in and closing and opening the outlets were done manually. 


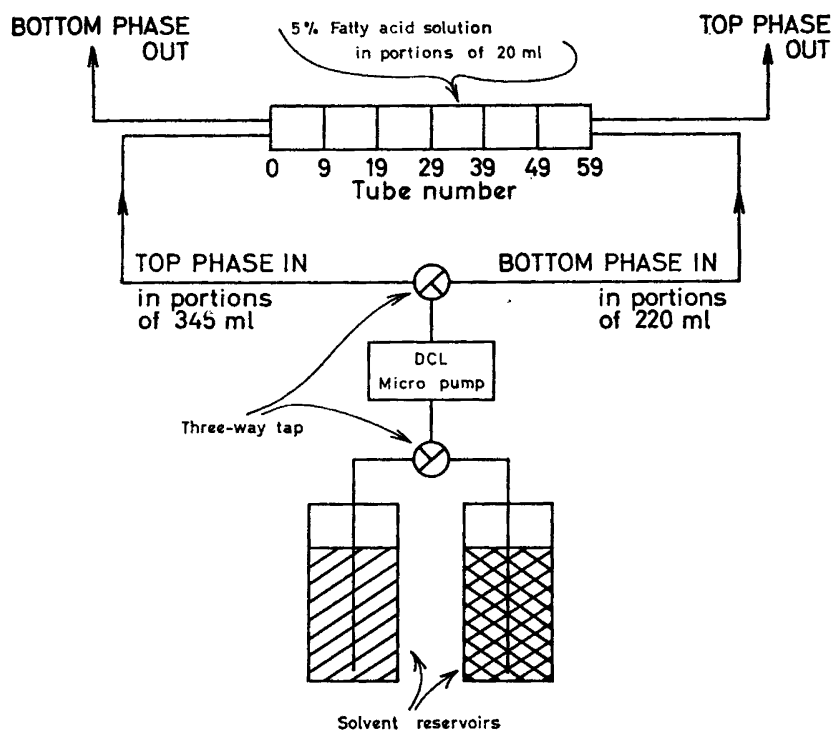

Fig. 2. Liquid-liquid extraction of linseed oil fatty acids first fractionated by crystallization as "acid" sodium soaps. Top phase (hexane) and bottom phase (methanol:dimethyl sulfoxide:water 75:20:5 v/v) were fed successively alternating in portions seen in the figure. Ratio of top to bottom phase $\left(v_{t} / v_{b}\right)$ was 1.57 and the flow rate $9 \mathrm{ml} / \mathrm{min}$.

Results. The emerging of the fatty acids in both effluent phases reached its maximum value after the ninth fractions and remained at a constant level thereafter. According to gas chromatographic analyses, the fatty acid compositions of the effluent fractions after the equilibrium had been reached were as follows $(\%)$ :

$\begin{array}{lccccc} & 16: 0 & 18: 2 & 18: 2 & 18: 3 & \begin{array}{c}\text { Unknown } \\ \text { substance }\end{array} \\ \text { Top phase } & 2.2 & 30.8 & 41.6 & 25.4 & - \\ \text { Bottom phase } & - & + & 3.2 & 96.8 & +\end{array}$

The fatty acid concentration of the effluent top and bottom phases were $0.095 \mathrm{~g} / 100 \mathrm{ml}$ and $0.228 \mathrm{~g} / 100 \mathrm{ml}$, respectively. Of the linolenic acid flowing out from the apparatus, $85.5 \%$ was thus recovered in the bottom phase.

\section{DISCUSSION}

In fat technology liquid-liquid extraction methods are used to separate oils or fatty acids into more and less saturated fractions. In the Pittsburgh Glass Plate Company process furfural served as a selective solvent, and it is used also when separating linoleic and linolenic acids from safflower and linseed oil acids in pilot plant scale. ${ }^{2,3}$

Acta Chem. Scand. 20 (1966) No. 6 
In a solvent system consisting of light petroleum: $95 \%$ methanol ${ }^{\mathbf{5}}$ distribution coefficients of $1.4,0.92$, and 0.72 are reported for oleic, linoleic, and linolenic acids; the separation factors are thus $1.21,1.30$, and 1.58. When comparing these to the separation factors obtained in the present work, it can be concluded that the addition of furfural to methanol markedly improves the selectivity of methanol. Unfortunately furfural is readily oxidized, which is a disadvantage of this solvent.

Will ${ }^{11}$ has suggested the use of dimethyl sulfoxide for the separation of fatty acids in countercurrent extraction. This solvent, like furfural, improved the selectivity of methanol in the present work. A disadvantage of the methanol:dimethyl sulfoxide mixture is, however, that the solubility of the upper phase (hexane) into the lower phase seems to increase greatly with increasing fatty acid concentration. This caused that the ratio of the phases in the distribution tubes did not stay constant during the process. For this reason the use of dimethyl sulfoxide alone without methanol would be more convenient, but it would make the procedure more complicated, since methanol has to be distilled off after the preliminary fractionation by crystallization. The use of acetonitrile instead of dimethyl sulfoxide might be a way of avoiding this difficulty. Acetonitrile:methanol:acetic acid mixture is used as a selective solvent in liquid-liquid extraction of fatty acids ${ }^{1}$ and acetonitrile was found very effective in the crystallization of linseed oil fatty acid as well. ${ }^{10}$ From linseed oil acids one crystallization at $-20^{\circ} \mathrm{C}$ gives concentrates containing over $70 \%$ linolenic acid, which is a better result than obtained by crystallization as "acid" sodium soaps at the same temperature. ${ }^{8,9}$ The simplest and the most efficient way to combine crystallization with liquid-liquid extraction would thus be to use acetonitrile in both separation procedures. In addition, this solvent is advantageous in that it is easily removed by distillation; thus the recovery of linolenic acid from the effluent presents no problems.

Effective separation of linolenic acid by countercurrent extraction can be reached with a small number of tubes by the principle used here. Theoretically the efficiency of 60 tubes corresponds to that of 400 tubes for a $99.7 \%$ yield, if the two components to be separated are symmetrically distributed around the middle tube of the row loaded at the beginning of the fractionation. The calculations made for the symmetrical distribution of linoleic acids do not apply in the procedure used here, since the increase in the fatty acid concentration before the equilibrium state is reached is not taken into consideration in them. A purity of more than $96 \%$ for linolenic acid in a $85 \%$ yield was, however, attained under the conditions of the present experiments. It should be remembered, however, that the purity of linolenic acid was here determined by gas chromatography only. Colored compounds, supposed to be oxidized and/or conjugated compounds, flowed out together with linolenic acid in the bottom phase, and for this reason gas chromatography alone was insufficient for determining the real purity of the linolenic acid.

It is concluded that combined crystallization and liquid-liquid extraction is an effective procedure for separating linolenic acid from linseed oil. In principle this method can be used for other materials, too, for separating for instance linoleic acid from soybean oil acids, although the procedures in this case are somewhat complicated, since two extractions are required, one for 
linoleic-linolenic and one for linoleic-oleic acids. From the technical point of view it is important that these methods as combined can in principle be used in large-scale work, too, for producing reasonable pure acids, and thus their use is dependent on economical factors only.

Acknowledgement. The authors wish to thank the United States Department of Agriculture, Agricultural Research Service, which partly financed this research.

\section{REFERENCES}

1. Ahrens, E. H. and Craig, L. C. J. Biol. Chem. 195 (1952) 299.

2. Beal, R. E. and Brekke, O. L. J. Am. Chemists' Soc. 36 (1959) 397.

3. Beal, R. E. et al. J. Am. Oil Chemists' Soc. 38 (1961) 525.

4. Butterfied, R. O., Dutton, H. J. and Schofield, C. R. Anal. Chem. 38 (1966) 86.

5. Davies, L. S. and Edwards, W. G. H. J. Sci. Food Agr. 2 (1952) 431.

6. Hietala, P. Ann. Acad. Sci. Fennicae, Ser. A II 1960 No. 100.

7. Sohns, V. E., Beal, R. E. and Griffin, E. L., Jr. J. Am. Oil Chemists' Soc. 40 (1963) 169.

8. Uksila, E. Ann. Acad. Sci. Fennicae, Ser. A II 1963 No. 119.

9. Uksila, E., Roine, P., Syväoja, E-L. and Alivaara, A. Acta Chem. Scand. 17 (1963) 2622.

10. Uksila, E. and Lehtinen, I. Acta Chem. Scand. 20 (1966) 1645.

11. Will, F. Anal. Chem. 33 (1961) 647.

Received March 9, 1966. 